\title{
New Constraints on Spatial Variations of the Fine Structure Constant from Clusters of Galaxies
}

\author{
Ivan De Martino ${ }^{1, *}$, Carlos J. A. P. Martins ${ }^{2,3}$, Harald Ebeling ${ }^{4}$ and Dale Kocevski ${ }^{5}$ \\ 1 Faculty of Science and Technology, Department of Theoretical Physics and History of Science, \\ University of the Basque Country (UPV/EHU), Barrio Sarriena s/n, 48940 Leioa, Spain \\ 2 Centro de Astrofisica da Universidade do Porto, Rua das Estrelas s/n, 4150-762 Porto, Portugal; \\ Carlos.Martins@astro.up.pt \\ 3 Instituto de Astrofísica e Ciências do Espaço, CAUP, Rua das Estrelas, 4150-762 Porto, Portugal \\ 4 Institute for Astronomy, University of Hawaii, Honolulu, HI 96822, USA; ebeling@ifa.hawaii.edu \\ 5 Department of Physics and Astronomy, Colby College, Waterville, ME 04901, USA; dale.kocevski@colby.edu \\ * Correspondence: ivan.demartino1983@gmail.com
}

Academic Editors: Mariusz P. Dabrowski, Manuel Krämer and Vincenzo Salzano

Received: 30 October 2016; Accepted: 12 December 2016; Published: 21 December 2016

\begin{abstract}
We have constrained the spatial variation of the fine structure constant using multi-frequency measurements of the thermal Sunyaev-Zeldovich effect of $618 \mathrm{X}$-ray selected clusters. Although our results are not competitive with the ones from quasar absorption lines, we improved by a factor 10 and $\sim 2.5$ previous results from Cosmic Microwave Background power spectrum and from galaxy clusters, respectively.
\end{abstract}

Keywords: cluster of galaxies; Cosmic Microwave Background; varying fine structure constant; Sunyaev-Zeldovich effect

\section{Introduction}

Recently, large observational studies of Quasar (QSO) absorption lines claimed that the fine structure constant could vary over the sky with a dipolar modulation [1-5]. The direction of such a dipole is comparable to the direction of other so-called anomalies and/or dipoles [6,7]. However, complementary analyses carried out on the Cosmic Microwave Background (CMB) power spectrum [8,9], and studying the effect of such modulation on the orbital motion of the major bodies of the solar system [10], do not report any detection. Since their sensitivity is, however, one thousand times worse than the one achieved with QSO data, there is a need to carry out tests with other observational datasets such as multi-frequency measurements of the thermal Sunyaev Zeldovich effect (TSZ, [11]) in cluster of galaxies.

The TSZ effect induces secondary anisotropies on the CMB power spectrum. Such anisotropies are usually expressed in terms of the Comptonization parameter $\left(Y_{c}\right)$ as $\Delta T_{T S Z}=T_{0} G(\tilde{v}) Y_{c}$, where $G(\tilde{v})$ is the TSZ spectral dependence, $Y_{c}$ is proportional to the integral along the line of sight of the pressure profile of the intra-cluster medium, and $T_{0}$ is the current value of the CMB black-body temperature. Moreover, $\tilde{v}=h v(z) / k_{B} T_{C M B}(z)$ is the reduced frequency, $k_{B}$ is the Boltzmann constant, $h$ is the Planck constant and $T_{C M B}(z)$ is the $\mathrm{CMB}$ black-body temperature at the cluster location.

In the concordance $\Lambda \mathrm{CDM}$ model, the CMB black-body temperature is $T_{C M B, s t d}(z)=T_{0}(1+z)$. Any departure from such behavior should imply an extension of the concordance model. In particular classes of models, where a scalar field is coupled to the Maxwell $F^{2}$ term in the matter Lagrangian, 
the photon number conservation is violated. Thus, the violation of the standard $T_{C M B}(z)$ law is coupled to the variation of the fine-structure as

$$
\frac{T_{C M B}(z)}{T_{0}} \sim(1+z)\left(1+\epsilon \frac{\Delta \alpha}{\alpha}\right)
$$

and their common origin implies that the coefficient $\epsilon$ is expected to be of order unity [12]; for example, in a somewhat simplistic adiabatic limit $\epsilon=1 / 4$. Thus, if one is able to determine $T_{C M B}(z)$ at the cluster location using multi-frequency measurements of the TSZ effect, then Equation (1) can be used as a phenomenological relation to observationally test the spatial variation of $\alpha$.

We apply the techniques developed in [13] to measure the TSZ emission at the location of 618 X-ray selected clusters. Then, we use such measurements to test the spatial variation of the fine structure constant. The article is organized as follows: in Section 2 we will describe the data; in Section 3 we will explain the methodology; in Section 4 we will illustrate and discuss our results; and finally, in Section 5, we will give our main conclusions.

\section{Data}

Galaxy clusters were selected from the ROSAT-ESO Flux Limited X-ray catalog (REFLEX, [14]), the extended Brightest Cluster Sample (eBCS, $[15,16])$ and the Clusters in the Zone of Avoidance (CIZA, [17]). The catalog lists 618 galaxy clusters for which all quantities of interest for our analysis are directly measured or derived: positions, spectroscopic redshifts, the central Comptonization parameter $\left(y_{c, 0}\right)$, the scale radius at which the mean overdensity of the cluster is 500 times the critical density $\left(r_{500}\right)$, and the corresponding angular size $\theta_{500}=r_{500} / d_{A}(z)$. Here, $d_{A}(z)$ is the angular diameter distance of each cluster. At the location of each cluster we measure the TSZ anisotropies using the Planck 2013 maps (Maps were originally released in a Healpix format with resolution $N_{\text {side }}=2048$ [18]. Data are available at http:/ / www.cosmos.esa.int/web/planck).

In March 2013, the Planck Collaboration made publicly available its nine Nominal maps covering the frequency channels from 30 to $857 \mathrm{GHz}$. Since the TSZ effect has a peculiar spectral dependence it can be distinguished from other components and reliably detected once the foreground emissions and the cosmological CMB signal are reduced. For that purpose, we apply a cleaning procedure, fully described in $[13,19,20]$, to the High Frequency Instrument data (100-857 GHz), since they have better resolution and lower instrumental noise than the Low Frequency Instrument data $(30-70 \mathrm{GHz})$. Before applying the cleaning procedure, we remove the intrinsic CMB monopole and dipole from the Nominal maps (We use the remove_dipole.pro facility of Healpix. It simultaneously performs a least square fit of the monopole and dipole on all the valid pixels and removes them from the map. More details can be found at http:/ / healpix.jpl.nasa.gov/html/subroutinesnode86.htm). The cleaning procedure returns, for each Planck frequency channel, a foreground cleaned patch centered at the position of each cluster in our catalog. Then, in each patch and for each frequency, we measure the average TSZ temperature $\left(\delta \bar{T} / T_{0}(v)\right)$ over discs with angular extent equal to $\theta_{500}$. Hereafter, the averaged quantities are always evaluated on discs of radius $\theta_{500}$ and indicated with a bar. To assign an error bar to each of the measurements, we carry out 1000 random simulations. In each one, we evaluate the mean temperature fluctuations in patches randomly placed out of the cluster positions and clean using the same procedure adopted for the real cluster population. Finally, we compute the correlation matrix between different frequencies $\left(C_{i j}\right)$

$$
C_{i j} \equiv C\left(v_{i}, v_{j}\right)=\frac{\left\langle\left[\delta \bar{T}\left(v_{i}\right)-\mu\left(v_{i}\right)\right]\left[\delta \bar{T}\left(v_{j}\right)-\mu\left(v_{j}\right)\right]\right\rangle}{\sigma\left(v_{i}\right) \sigma\left(v_{j}\right)},
$$

where the average was computed over all simulations, $\mu\left(v_{i}\right)=\left\langle\delta \bar{T}\left(v_{i}\right)\right\rangle$, and $\sigma\left(v_{i}\right)=\left\langle\left[\delta \bar{T}\left(v_{i}\right)-\mu\left(v_{i}\right)\right]^{2}\right\rangle^{1 / 2}$. The square roots of the diagonal elements of the correlation matrix are the error bars associated to the TSZ temperature anisotropies of each cluster. 


\section{Methodology}

To estimate the CMB temperature at the cluster location, as well as to constrain the spatial variations of the fine structure constant, we use a Monte Carlo Markov Chain (MCMC) technique to explore the corresponding relevant parameter space. Our pipelines employ the Metropolis-Hastings sampling algorithm [21,22] with different (randomly set) starting points, and the Gelman-Rubin criteria [23] to test the convergence and the mixing of each chain. Specifically, we compute the ratio of the variances in the target distribution, and require it to be $\mathcal{R}<1.03$ (mathematical definitions are given in Section 3.2 of [24]). We run four chains that stop when they satisfy the criteria and contain at least 30,000 steps. Finally the chains are merged, and the expectation value of the 1D marginalized likelihood distribution is computed using all the steps.

First we need to estimate the CMB temperature at the cluster location. Having measured for each galaxy cluster the averaged TSZ emission as a function of frequency, we predict the theoretical counterpart at the same aperture $\Delta \bar{T}\left(\mathbf{p}, v_{i}\right) / T_{0}=G\left(v_{i}, T_{C M B}(z)\right) \bar{Y}_{c}$, where $\mathbf{p}=\left[T_{C M B}(z), \bar{Y}_{c}\right]$ are the free parameters of the model, and $\bar{Y}_{\mathcal{C}}$ is the averaged Comptonization parameter. Finally, we compute the likelihood $-2 \log \mathcal{L}=\chi^{2}(\mathbf{p})$ as

$$
-2 \log \mathcal{L}=\chi^{2}(\mathbf{p})=\sum_{i=0}^{N} \Sigma_{j=0}^{N} \Delta \bar{T}_{i}(\mathbf{p}) C_{i j}^{-1} \Delta \bar{T}_{j}(\mathbf{p}),
$$

where $\Delta \bar{T}_{i}(\mathbf{p}) \equiv \frac{\Delta \bar{T}\left(\mathbf{p}, v_{i}\right)}{T_{0}}-\frac{\delta \bar{T}\left(v_{i}\right)}{T_{0}}$, and $N=4$ is the number of data points (the four frequencies). Once our simulations reached the convergence criteria, we estimated the CMB temperature for each individual cluster, and its $68 \%$ of confidence level error bar, from the 1D marginalized likelihood distribution. See [25] for a full description of the methodology. These measurements are used to estimate the $\alpha$-anisotropies as

$$
\frac{\Delta \alpha}{\alpha}=\epsilon^{-1}\left(1-\frac{T_{C M B}(z)}{T_{0}(1+z)}\right) .
$$

Finally, we probe 3 different models allowing for a monopole $(m)$ amplitude plus dipole $(d)$ variation. Specifically, the first model assumes the functional form defined in [1] to fit quasar data (Model 1)

$$
\frac{\Delta \alpha}{\alpha}=m+d \cos (\Theta),
$$

where $\Theta$ is the angle on the sky between the line of sight of each cluster and the best fit dipole direction. Then, we fit the model used in [26] to the cluster data. Such a model (Model 2) allows a variation with the $\Lambda$ CDM look-back time, $r(z)=\int \frac{d z^{\prime}}{H\left(z^{\prime}\right)}$ :

$$
\frac{\Delta \alpha}{\alpha}=m+d r(z) \cos (\Theta) .
$$

Finally, to explore the possibility of an intrinsic dipole, we also try to fit the following relation to the CMB temperature measurements (Model 3)

$$
\frac{\Delta T}{T_{0}(1+z)}=m+d \cos (\Theta) .
$$

For each model we analyze four different configurations of the parameter space: (A) $m=0$ for the Model 1 and 2, and unity for Model 3, the direction of dipole is fixed to the one from QSO [1], while the amplitude vary in the interval $[-1,1]$; (B) the direction is still fixed, but we also vary the monopole in the intervals $[-1,1]$ for Model 1 and 2, and [0,2] for Model 3; (C) and (D) we repeat the configurations of (A) and (B) but we also vary the dipole direction over the whole sky. 


\section{Results and Discussion}

Our results show that the monopole and dipole amplitudes are always compatible with their expected valued in standard cosmology within $1.5 \sigma$. The best constraints are: $m=0.006 \pm 0.004$ and $d=-0.008 \pm 0.009$ for Model 1 (B), and $d=-0.003 \pm 0.003 \mathrm{GLyr}^{-1}$ and $m=0.006 \pm 0.005$ for Model 2 (A) and (B), respectively. The direction of the "possible" dipole is always compatible with the one predicted from QSO in [1], but when they are varied the constraints on the monopole and dipole amplitudes are degraded since the number of free parameters is increased. Nevertheless, our best fit directions are always compatible with the one from QSO data [1,2] and other CMB anomalies [6,7], while they are several degrees away from the directions of the intrinsic CMB and the Dark Flow dipoles, from the kinetic CMB asymmetry, and from the CMB cold spot location [27-39]. All results are summarized in the Table 1 and, for easier comparison, the anomalies directions are plotted in Figure 1.

Since our analysis shows that the possible dipole direction coincides with the one obtained from QSO [1] and dark energy models [6,7], it could be interpreted as the fact that such dipoles, if confirmed with more extended and independent analysis, could represent signature of the failure of the $\Lambda$ CDM model in favor of varying constant models or extended theories of gravity. Finally let us remark that, although we are not competitive with the QSO constraint from [1], our best fits for Model 2 provide a precision improvement of a factor $\sim 2.5$, while being compatible with the results in [26]. For Model 1, we improve on the previous constraints in $[8,9]$ by a factor $\sim 10$. The improvement is mainly due to the fact that previous analyses, based on the CMB power spectrum, need to fit not only all "standard" parameters of the cosmological model, but also the additional ones related to the variation of fundamental constants; as a consequence, the resulting constraints were less tight. 

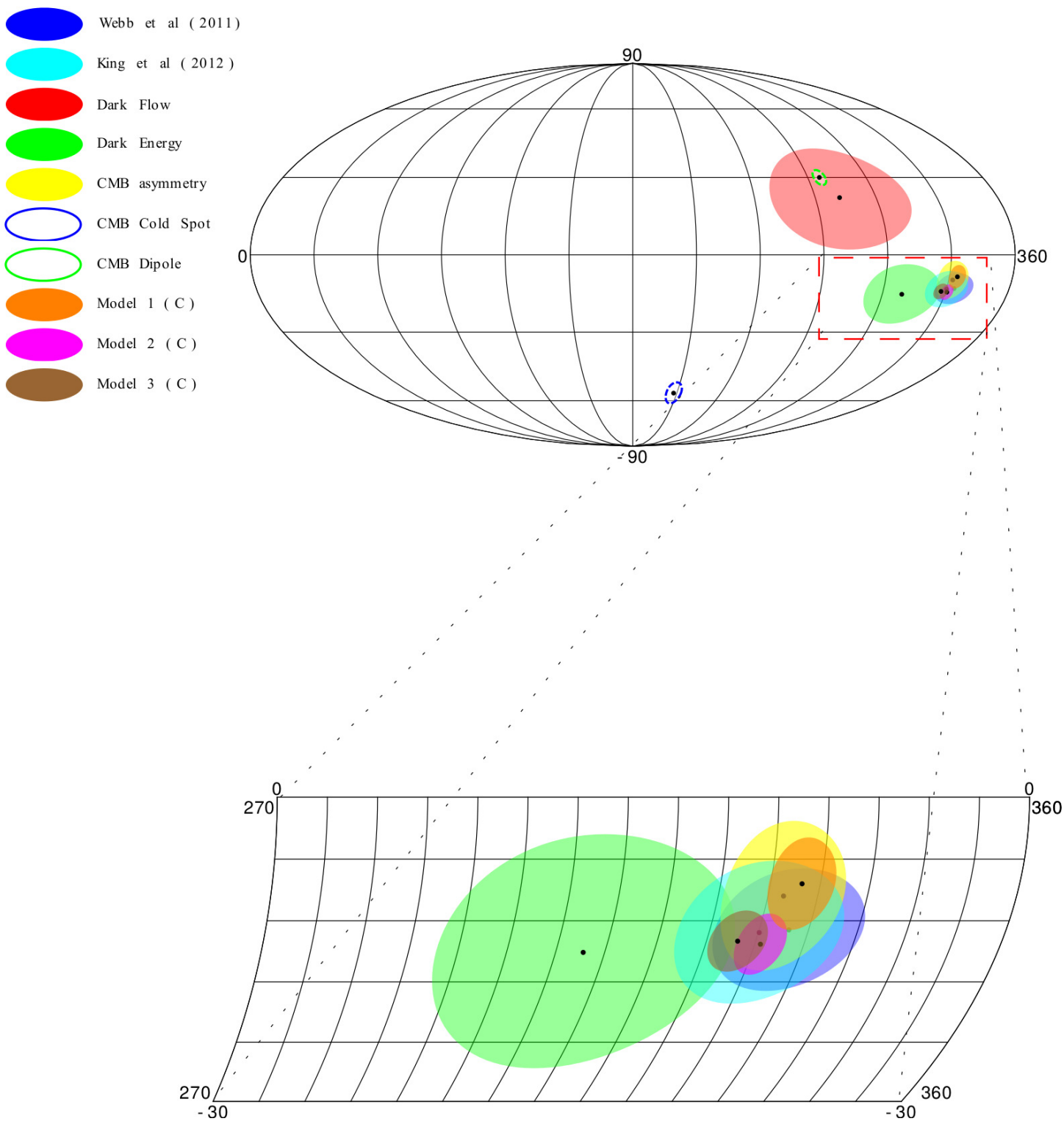

Figure 1. Directions in galactic coordinates for the spatial variation of the fine structure constant from [1,2] in blue and cyan, respectively, for the Dark Energy dipoles from [6] in green, Dark Flow direction from [27,30-33] in red, for the Cosmic Microwave Background (CMB) asymmetry from [7] in yellow, and for our results from the analysis (C) of models 1,2 and 3 in orange, magenta and brown, respectively. Finally, we also indicate in blue and green dashed circles the direction of the Cold Spot anomaly and the intrinsic CMB dipole, respectively $[28,29,34]$.

\section{Conclusions}

We have considered a model in which the variation of the fine structure constant is coupled to the variation of the CMB temperature. Using Equation (1), we have constrained the spatial variation of the fine structure constant using the Planck 2013 Nominal maps and a X-ray selected cluster catalog. First, we have cleaned the Planck maps and extracted the CMB temperature at the location of $618 \mathrm{X}$-ray selected clusters using the TSZ multi-frequency measurements. Then, we have carried out a statistical analysis to test three models allowing for monopole and dipole amplitudes, and describing both spatial variations of $\Delta \alpha / \alpha$ and of $T_{C M B}$ itself. All results of our analysis are summarized in Table 1. 
Table 1. Results of the analysis for Models 1, 2 and 3 from [25].

\begin{tabular}{cccccc}
\hline & & $m$ & $d$ & RA $\left(^{\circ}\right)$ & DEC $\left(^{\circ}\right)$ \\
\hline \multirow{5}{*}{ Model 1 } & (A) & 0.0 & $-0.002 \pm 0.008$ & 261.0 & -58.0 \\
& (B) & $0.006 \pm 0.004$ & $-0.008 \pm 0.009$ & 261.0 & -58.0 \\
& (C) & 0.0 & $-0.030 \pm 0.020$ & $255.1 \pm 3.8$ & $-63.2 \pm 2.6$ \\
& (D) & $0.021 \pm 0.029$ & $-0.030 \pm 0.014$ & $255.9 \pm 4.2$ & $-55.3 \pm 5.8$ \\
\hline \multirow{5}{*}{ Model 2 } & (A) & 0.0 & $-0.003 \pm 0.003 \mathrm{GLyr}^{-1}$ & 261.0 & -58.0 \\
& (B) & $0.006 \pm 0.005$ & $-0.003 \pm 0.005 \mathrm{GLyr}^{-1}$ & 261.0 & -58.0 \\
& (C) & 0.0 & $-0.042 \pm 0.049 \mathrm{GLyr}^{-1}$ & $261.6 \pm 16.1$ & $-61.3 \pm 2.7$ \\
& (D) & $0.019 \pm 0.011$ & $-0.027 \pm 0.051 \mathrm{GLyr}^{-1}$ & $245.0 \pm 12.9$ & $-56.0 \pm 3.8$ \\
\hline \multirow{5}{*}{ Model 3 } & (A) & 1.0 & $-0.010 \pm 0.008$ & 261.0 & -58.0 \\
& (B) & $1.001 \pm 0.002$ & $-0.003 \pm 0.002$ & 261.0 & -58.0 \\
& (C) & 1.0 & $-0.020 \pm 0.015$ & $258.0 \pm 1.2$ & $-64.0 \pm 1.1$ \\
& (D) & $1.000 \pm 0.0001$ & $-0.018 \pm 0.015$ & $258.4 \pm 1.9$ & $-64.3 \pm 2.6$ \\
\hline
\end{tabular}

Although at the present time galaxy clusters are not competitive with high-resolution spectroscopic measurements in absorption systems along the line-of-sight of bright quasars, our analysis was improved by an order of magnitude previous constraints by the Planck Collaboration [8] and by a factor $\sim 2.5$ the results from Galli (2013) [26].

Acknowledgments: I.D.M. acknowledges the financial support from the University of the Basque Country UPV/EHU under the program "Convocatoria de contratación para la especialización de personal investigador doctor en la UPV/EHU 2015", from the Spanish Ministry of Economy and Competitiveness through research project FIS2014-57956-P (comprising FEDER funds) and from the Basque Government grant IT956-16 for the GIC research group. This article is based upon work from COST Action CA1511 Cosmology and Astrophysics Network for Theoretical Advances and Training Actions (CANTATA), supported by COST (European Cooperation in Science and Technology). This research was conducted in the context of project PTDC/FIS/111725/2009 (FCT, Portugal), with additional support from grant UID/FIS/04434/2013. C.J.M. is also supported by an FCT Research Professorship, contract reference IF/00064/2012, funded by FCT/MCTES (Portugal) and POPH/FSE (EC). C.J.M. thanks the Galileo Galilei Institute for Theoretical Physics for the hospitality and the INFN for partial support during the completion of this work.

Author Contributions: I.D.M. and C.J.M. conceived the project and wrote the paper; I.D.M. performed the data analysis; H.E. and D.K. provided the X-ray cluster catalog.

Conflicts of Interest: The authors declare no conflict of interest.

\section{References}

1. Webb, J.K.; King, J.A.; Murphy, M.T.; Flambaum, V.V.; Carswell, R.F.; Bainbridge, M.B. Indications of a Spatial Variation of the Fine Structure Constant. Phys. Rev. Lett. 2011, 107, 191101.

2. King, J.A.; Webb, J.K.; Murphy, M.T.; Flambaum, V.V.; Carswell, R.F.; Bainbridge, M.B.; Wilczynska, M.R.; Koch, F.E. Spatial variation in the fine-structure constant-New results from VLT/UVES. Mon. Not. R. Astron. Soc. 2012, 422, 3370-3414.

3. Berengut, J.C.; Flambaum, V.V.; King, J.A.; Curran, S.J.; Webb, J.K. Is there further evidence for spatial variation of fundamental constants? Phys. Rev. D 2011, 83, 123506.

4. Berengut, J.C.; Kava, E.M.; Flambaum, V.V. Is there a spatial gradient in values of the fine-structure constant? A reanalysis of the results. Astron. Astrophys. 2012, 542, A118.

5. Pinho, A.M.M.; Martins, C.J.A.P. Updated constraints on spatial variations of the fine-structure constant. Phys. Lett. B 2016, 756, 121-125.

6. Mariano, A.; Perivolaropoulos, L. Is there correlation between fine structure and dark energy cosmic dipoles? Phys. Rev. D 2012, 86, 083517.

7. Mariano, A.; Perivolaropoulos, L. CMB maximum temperature asymmetry axis: Alignment with other cosmic asymmetries. Phys. Rev. D 2013, 87, 043511.

8. Planck Collaboration. Planck intermediate results. XXIV. Constraints on variation of fundamental constants. Astron. Astrophys. 2015, 580, A22. 
9. O’Bryan, J.; Smidt, J.; De Bernardis, F.; Cooray, A. Constraints on Spatial Variations in the Fine-structure Constant from Planck. Astrophys. J. 2015, 798, 18.

10. Iorio, L. Orbital effects of spatial variations of fundamental coupling constants. Mon. Not. R. Astron. Soc. 2011, 417, 2392-2400.

11. Sunyaev, R.A.; Zeldovich, Y.B. The Observations of Relic Radiation as a Test of the Nature of X-Ray Radiation from the Clusters of Galaxies. Comments Astrophys. Space Phys. 1972, 4, 173-178.

12. Avgoustidis, A.; Martins, C.J.A.P.; Monteiro, A.M.R.V.L.; Vielzeufb, P.E.; Luzzi, G. Cosmological effects of scalar-photon couplings: Dark energy and varying- $\alpha$ Models. J. Cosmol. Astropart. Phys. 2014, $2014,062$.

13. De Martino, I.; Génova-Santos, R.T.; Atrio-Barandela, F.; Ebeling, H.; Kashlinsky, A.; Kocevski, D.; Martins, C.J.A.P. Constraining the Redshift Evolution of the Cosmic Microwave Background Blackbody Temperature with PLANCK Data. Astrophys. J. 2015, 808, 128.

14. Böhringer, H.; Schuecker, P.; Guzzo, L.; Collins, C.A.; Voges, W.; Cruddace, R.G.; Ortiz-Gil, A.; Chincarini, G.; De Grandi, S.; Edge, A.C.; et al. The ROSAT-ESO Flux Limited X-ray (REFLEX) Galaxy cluster survey. V. The cluster catalogue. Astron. Astrophys. 2004, 425, 367-383.

15. Ebeling, H.; Edge, A.C.; Böhringer, H.; Allen, S.W.; Crawford, C.S.; Fabian, A.C.; Voges, W.; Huchra, J.P. The ROSAT Brightest Cluster Sample-I. The compilation of the sample and the cluster $\log N-\log S$ distribution. Mon. Not. R. Astron. Soc. 1998, 301, 881-914.

16. Ebeling, H.; Edge, A.C.; Allen, S.W.; Crawford, C.S.; Fabian, A.C.; Huchra, J.P. The ROSAT Brightest Cluster Sample-IV. The extended sample. Mon. Not. R. Astron. Soc. 2000, 318, 333-340.

17. Ebeling, H.; Mullis, C.R.; Tully, R.B. A Systematic X-Ray Search for Clusters of Galaxies behind the Milky Way. Astrophys. J. 2002, 580, 774-788.

18. Górski, K.M.; Hivon, E.; Banday, A.J.; Wandelt, B.D.; Hansen, F.K.; Reinecke, M.; Bartelmann, M. HEALPix: A Framework for High-Resolution Discretization and Fast Analysis of Data Distributed on the Sphere. Astrophys. J. 2005, 622, 759-771.

19. De Martino, I.; Atrio-Barandela, F. SZ/X-ray scaling relations using X-ray data and Planck Nominal maps. Mon. Not. R. Astron. Soc. 2016, 461, 3222-3232.

20. De Martino, I. A $f(R)$-gravity model of the Sunyaev-Zeldovich profile of the Coma cluster compatible with Planck data. Phys. Rev. D 2016, 93, 124043.

21. Hastings, W.K. Monte Carlo Sampling Methods Using Markov Chains and Their Applications. Biometrika 1970, 57, 97-109.

22. Metropolis, N.; Rosenbluth, A.W.; Rosenbluth, M.N.; Teller, A.H.; Teller, E. Equation of State Calculations by Fast Computing Machines. J. Chem. Phys. 1953, 21, 1087-1092.

23. Gelman, A.; Rubin, D.B. Inference from Iterative Simulation Using Multiple Sequences. Stat. Sci. 1992, 7, 457-472.

24. Verde, L.; Peiris, H.V.; Spergel, D.N.; Nolta, M.R.; Bennett, C.L.; Halpern, M.; Hinshaw, G.; Jarosik, N.; Kogut, A.; Limon, M.; et al. First Year Wilkinson Microwave Anisotropy Probe (WMAP) Observations: Parameter Estimation Methodology. Astrophys. J. Suppl. Ser. 2003, 148, 195-211.

25. De Martino, I.; Martins, C.J.A.P.; Ebeling, H.; Kocevski, D. Constraining spatial variations of the fine structure constant using clusters of galaxies and Planck data. Phys. Review D 2016, 94, 083008.

26. Galli, S. Clusters of galaxies and variation of the fine structure constant. Phys. Rev. D 2013, 87, 123516.

27. Kashlinsky, A.; Atrio-Barandela, F.; Kocevski, D.; Ebeling, H. A Measurement of Large-Scale Peculiar Velocities of Clusters of Galaxies: Results and Cosmological Implications. Astrophys. J. Lett. 2008, 686, L49.

28. Kogut, A.; Lineweaver, C.; Smoot, G.F.; Bennett, C.L.; Banday, A.; Boggess, N.W.; Cheng, E.S.; de Amici, G.; Fixsen, D.J.; Hinshaw, G.; et al. Dipole Anisotropy in the COBE Differential Microwave Radiometers First-Year Sky Maps. Astrophys. J. 1993, 419, 1-6.

29. Vielva, P.; Martinez-Gonzalez, E.; Barreiro, R.B.; Sanz, J.L.; Cayon, L. Detection of non-gaussianity in the Wilkinson microwave anisotropy probe first-year data using spherical wavelets. Astrophys. J. 2004, 609, $22-34$.

30. Kashlinsky, A.; Atrio-Barandela, F.; Ebeling, H.; Edge, A.; Kocevski, D. A New Measurement of the Bulk Flow of X-Ray Luminous Clusters of Galaxies. Astrophys. J. Lett. 2010, 712, L81.

31. Kashlinsky, A.; Atrio-Barandela, F.; Ebeling, H. Measuring the Dark Flow with Public X-ray Cluster Data. Astrophys. J. 2011, 732, 1. 
32. Kashlinsky, A.; Atrio-Barandela, F.; Ebeling, H. Measuring bulk motion of X-ray clusters via the kinematic Sunyaev-Zeldovich effect: Summarizing the "dark flow" evidence and its implications. arXiv 2012, arXiv:1202.0717v1.

33. Atrio-Barandela, F.; Kashlinsky, A.; Ebeling, H.; Fixsen, D.J.; Kocevski, D. Probing the Dark Flow Signal in WMAP 9 -Year and Planck Cosmic Microwave Background Maps. Astrophys. J. 2015, 810, 143.

34. Ade, P.A.; Aghanim, N.; Armitage-Caplan, C.; Arnaud, M.; Ashdown, M.; Atrio-Barandela, F.; Aumont, J.; Baccigalupi, C.; Banday, A.J.; Barreiro, R.B.; et al. Planck 2013 results. XXIII. Isotropy and statistics of the CMB. Astron. Astrophys. 2014, 571, A23.

35. Naselsky, P.; Zhao, W.; Kim, J.; Chen, S. Is the Cosmic Microwave Background Asymmetry due to the Kinematic Dipole? Astrophys. J. 2012, 749, 31.

36. Zhao, W.; Wu, P.X.; Zhang, Y. Anisotropy of Cosmic Acceleration. Int. J. Mod. Phys. D 2013, 22, 1350060.

37. Zhao, W. Directional dependence of CMB parity asymmetry. Phys. Rev. D 2014, 89, 023010.

38. Zhao, W.; Santos, L. Preferred axis in cosmology. Universe 2016, 3, 9-33.

39. Cheng, C.; Zhao, W.; Huang, Q.-G.; Santos, L. Preferred axis of CMB parity asymmetry in the masked maps. Phys. Lett. B 2016, 757, 445-453.

(C) 2016 by the authors; licensee MDPI, Basel, Switzerland. This article is an open access article distributed under the terms and conditions of the Creative Commons Attribution (CC-BY) license (http://creativecommons.org/licenses/by/4.0/). 